\title{
Sentiment analysis of Twitter posts related to the COVID-19 vaccines
}

\author{
Noralhuda N. Alabid ${ }^{1}$, Zainab Dalaf Katheeth ${ }^{2}$ \\ ${ }^{1}$ Department of Computer Science, Faculty of Education, University of Kufa, Najaf, Iraq \\ ${ }^{2}$ Department of Computer Science, Faculty of Computer Science and Mathematics, University of Kufa, Najaf, Iraq
}

\begin{tabular}{|c|c|}
\hline Article Info & ABSTRACT \\
\hline & \multirow{9}{*}{$\begin{array}{l}\text { A real threat to the people of the world has appeared as a result of the spread } \\
\text { of the Coronavirus disease of } 2019 \text { (COVID-19) disease. A lot of scientific } \\
\text { and financial support has been made to devote vaccines capable of ending } \\
\text { this epidemic. However, these vaccines have become a subject of debate } \\
\text { between individuals, as some people tend to support taking vaccines and } \\
\text { others rejecting them. This paper aims to create a framework model to } \\
\text { classify the sentiment and opinions of individuals that published in Twitter } \\
\text { regarding the COVID-19 vaccines. Identify those opinions can help public } \\
\text { health institutions to know public opinions and direct their efforts towards } \\
\text { promoting taking vaccinations. Two of the machines learning classification } \\
\text { models which are the support vector machine (SVM) and naive Bayes (NB) } \\
\text { classifier are applied here. Other pre-processing methods were applied as } \\
\text { well to filter unstructured tweets. }\end{array}$} \\
\hline Received Aug 16, 2021 & \\
\hline Revised Oct 21, 2021 & \\
\hline Accepted Oct 27, 2021 & \\
\hline Keywords: & \\
\hline COVID-19 & \\
\hline Data analytics & \\
\hline Sentiment analysis & \\
\hline Text mining & \\
\hline
\end{tabular}

This is an open access article under the CC BY-SA license.

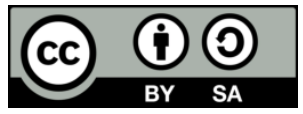

\author{
Corresponding Author: \\ Noralhuda N. Alabid \\ Department of Computer Science, Faculty of Education \\ University of Kufa \\ Najaf, Iraq \\ Email: noralhudan.hadi@uokufa.edu.iq
}

\section{INTRODUCTION}

There has been a lot of global effort to evolve and test an effective Coronavirus disease of 2019 (COVID-19) vaccine since the outbreak of the pandemic. Although preventive measures have contributed to limit the spread of this disease, hopes are pinned on finding an effective and sustainable vaccine to end this epidemic. Encouraging public for vaccination is critical to end COVID-19 pandemic. According to [1] over $60-80 \%$ of populations have to be vaccinated to reach some level of herd immunity. Also, realizing emotion is considered one of the essential aspects of building a successful life and growing human relationships. Furthermore, understanding others' thoughts has an effect on acquiring knowledge and making decisions [2]. Therefore, it is necessary to know public opinion about vaccination and their inclination to receive it. Social media is widespread platform that are used to discuss many topics. Spreading COVID-19 and receiving vaccinations is one of these topic [3]. Information obtained from social media may encourage individual to take vaccines or refuse them. The confidence of individuals in science and its role in solving this crisis can be destroyed by false information [4], which will affect the level of vaccination [5]-[7].

Obtained opinions of individuals about this topic is a challenging mission. Statistical processing of such huge information obtained from survey mostly loses the ability to give reliable results. Thus, robotic classifier for analysing opinions could be more practical. Using data from Twitter that consider one of the most used social media outlets can be more illustrative of real opinions [4]. 
Various aspects have to be considered for building a robust and accurate classifier. In most online comments, people uses irregular language to announce their opinions. Furthermore, these comments and posts contain various language errors, such as in grammars and word spelling [8], [9]. This obstacle opens challenges for analyzing and interpreting human language [10]. Text mining or sentiment analysis [11] appears to be fully grasp the automatic processing of natural language (NLP) [9], [12]. Yang et al. in [11] believed that sentiment analysis is aimed to analyse users' comments on the Internet in order to identify the underlying emotional information. According to [12], sentiment analysis is a process of analysis, processing, extrapolation, and conclusion of individual texts with an emotional color. Generally, sentiment analysis classifies opinions into positive and negative attitude [13]. This paper is organized: Section 1 provides an introduction and about the existing research. Section 2 presents a brief background and related work. Section 3 explains the framework that is developed for sentiment analysis. Section 4 highlights the analysis results. Finally, the conclusion is presented in the last section.

\section{RELATED WORK}

Several researches and different processes are performed in the field of sentiment analysis. Authors in [14], [15] used unsupervised learning algorithms to calculate the average semantic orientation of texts. In the article of [16], authors defined the feature of a product based on latent semantic analysis (LSA). Also, they used a statistical approach to determine opinion phrases. Authors in [17] stemed on adjective opinion words to extract information. They collected textual data from the web pages. Then, they manually labelled adjective words and use a comparative sentence to distinguish between positive and negative phrases. In [18], the polarity of sentences is identified based on baseline and support vector machines (SVM) methods. They extracted the polarity of overall documents for certain words by detecting the sentiment of dictionaries and classifying them based on textual data information. Khan and Baharudin [19] proposed a method which used SentiWordNet to classify words as a positive, negative, or neutral phrase. Authors in [20] used each of machine-learning techniques and cosine similarity to create a scheme for opinion classification. They suggested pre-processing the web data to improve the structure of textual data. Others researches used various techniques in sentiment analysis using method of neural network [21], [22], data mining [23], and artificial intelligence [24].

Some studies provide a simple descriptive analysis of Twitter data related vaccines to estimate individual's opinions toward vaccination [25], define prevailing opinions [26], track discussion societies on vaccination [27], determine patterns of vaccine [28]. A study examined the increasing activity in Twitter for anti-vaccination group and its result confirmed the effectiveness of these activities in refusing vaccinations [7]. Another study showed that the reluctance to take the vaccine is due to the negative impression taken from Twitter bots [28]. Generally, public opinion on vaccines regarding COIVD-19 is unclear. Identifying the public opinion on COVID-19 vaccines is of great significance as a rejection case against vaccination, which could contribute to widespread pandemic from a public health view.

Some have focused on distinguishing emotional expressions [29]-[32]. Others [33]-[37] have based on determining the polarity of the reviews whether these opinions are positive, negative, or neutral. In fact, the emotion detection task and polarity detection task are associated with each other, and in most cases, detection of the emotion represents a sub-function from the detection of the polarity. For the more advanced analysis task, polarity detection can be used as a subtask to classify customers' reviews whether they like or dislike services [38], [39]. This helps in judging the quality of the products [2], [5], [40], [41].

The influential sentimental analysis models also need to identify the topic of a review. This can help to improve the performance. For example, analyzing texts and ignoring the topic section can lead to obtain irrelevant emotional information and mislead the polarity analysis of the main topic. In addition, the topic of a review may discuss multiple subjects which are attractive for users. In addition, some topics may discuss multiple subjects which are attractive for users as well. Therefore, it is necessary to define topics [2]. Other features such as subjective and objective opinions classifications [42], aspect extraction [43], concept detection, gender of users [44] and topic recognition [45]. Also, they have their effects on the validation of the sentiment analysis models.

The increasing numbers of devices, which are used to post reviews, have an effect on creating different formats of information such as texts, audio files, and video material. Different activities can be utilized to collect the affective information such as analysis face expressions, physical interaction, the gaining impression from the background of videos and images [46], [47]. All of these data resources have to add to an integrated SA model [2]. According to the data of [35], it is possible to combine phonetics and linguistics to obtain information and assess opinions. Authors in [48] were indeed able to combined phonetics, linguistics, and videos to analyze information and determine opinion polarity. However, the manual transcript stills the main source of information to implement sentiment classification. 
A natural language is a tool used to declare human ideas. Mostly, these ideas contain complex knowledge and expressions. Natural languages do not follow restricted rules as with artificial and programming languages. Thus, natural languages can contain unpredictable difficulties and formats. Many aspects are needed to be considered when there is a need to create the model of sentiment analysis such as extracting the meaning of negations, emotional words, understanding the meaning of the emoji symbols, and others. Meanwhile, text processing technologies can be utilized to remove useless data, which lead to reduce the processing time and improve the efficiency of sentiment analysis.

\section{SENTIMENT ANALYSIS FRAMEWORK}

\subsection{Collecting the dataset}

To collect Twitter posts related to the topic of COVID-19 vaccination, we utilized Twitter application programming interface (API) in Python programming language to pull tweets [49], [50]. A combination of keywords "Corona", "COVID-19"," Coronavirus", and "vaccines" are used to retrieve tweets post in January 2021. The data was taken from English-speaker from the countries: the United Kingdom and India. In total, this data is about $(15,000)$ tweets. There was a study to create a model of real time sentiment analysis [51]. However, Table 1 shows some of the retrieval tweets for the proposal model. It is noted that positives tweets are higher average than negative or neutral tweets. Figure 1 shows the proportion of tweets in each class. Figure 2 displays some examples from the training data set that included all of the three labels.

Table 1. Training dataset statistics

\begin{tabular}{cc}
\hline Total number of tweets & 15030 \\
\hline Positive tweets & $49 \%$ \\
Negative tweet & $30 \%$ \\
Neutral tweets & $21 \%$ \\
\hline
\end{tabular}

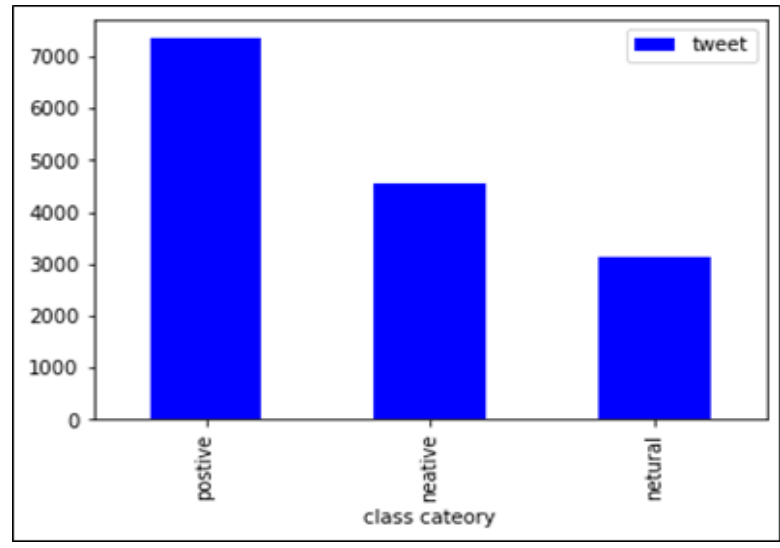

Figure1. Polarity sentiment distribution of tweets

No tweet label
1 Hysteria surrounding \#coronavirus NZ daycare renetural
2 Thank you @TheOnion for dragging all of us und Postive
3 \#avetmissdone is catching on faster than the \#ccPostive
4 They just said \#Tonysnell was back from the flu..negative
5 Forget locking them up on an island to die negative
6 Please keep posted!?????? \#CoronaVirus \#Gen netural
7 What did Richard Jefferson say? ?? \#coronaviru netural
8 When that coronavirus says "Ni Hao Ma" me get negative

Figure 2. Examples from collected tweets

\subsection{Labelling the dataset}

A machine learning algorithm (ML) is a two-step process, training, and testing dataset. Thus, we need to label some of the tweets with real opinions to be used in the training stage. Labelling the dataset has 
been done manually by using the human in the loop (HITL) services. We used three terms to annotate the polarity of tweets toward vaccine: positive, negative and, neutral. We added a certain condition in order to accept the selected label by users. This condition determines the compatibility in the opinion of both the user and the author through the labelling process. Otherwise, it is recommended that this tweet should be labelled by a third evaluator, and the final result should be determined by the majority of votes.

\subsection{Pre-processing dataset}

Pre-processing is an essential phase in texts mining [52]. It could be applied to texts for omitting unwanted terms such as punctuation, emails, numbers, and links. For that, natural language processing (NLP) techniques offer significant services. These techniques are used to reduce the size of textual data [53] and transfers texts from their original format to another reduced structure. Also, we used these techniques for normalizing/stemming, filtering the unwanted words, and tokenization. The scenario of pre-processing methods that were implemented for this work is: first, each tweet is split to obtain separated tokens. There are many ways that could be used to split words and not just depend on whitespace. For this, the comma, semicolon, and colon are also used for segmentation. Then, the following pre-processing technique was omitting unwanted words. These words are diverse that could be auxiliary verbs or negation terms. In sentiment analysis, the negation words are important to determine the polarity of text. Therefore, we built a special dictionary of English words that can be used to remove the useless words and keep the negation clauses. Finally, the stemming tokens technique was applied by using a specific function available in the NLP library.

\subsection{Building model}

We implemented two kinds of machine learning (ML) classifiers to classify tweets into positive, negative, and neutral expressions. We used two of the most influential classifiers which are the naive Bayes (NB) classifier and SVM classifier. Different pre-processing techniques are applied along with each classifier to examine the main factors that cause those differences in performance between these classifiers. Figure 3 shows the proposed scheme.

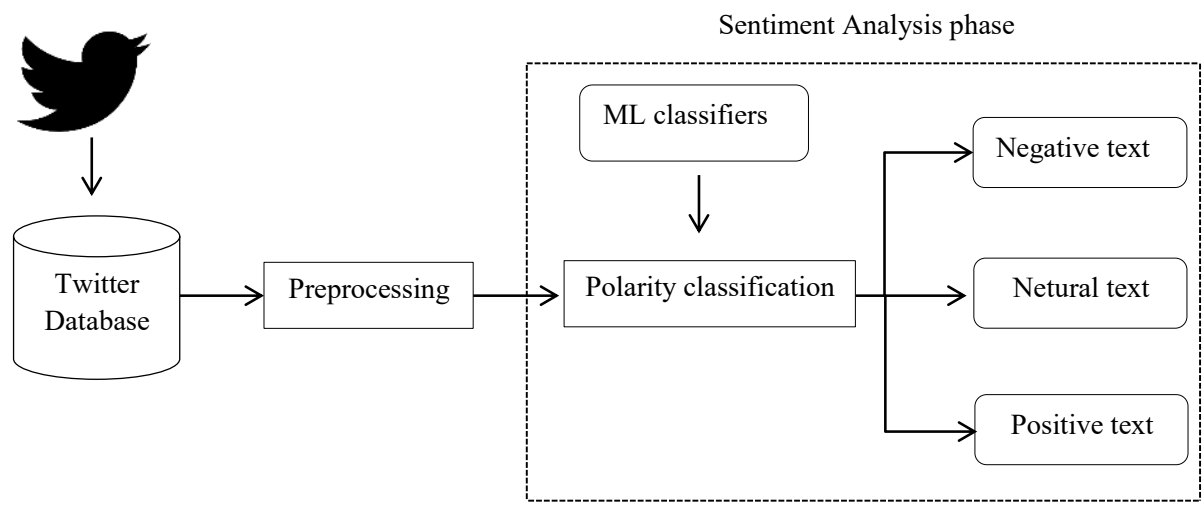

Figure 3. Outline of sentiment analysis scheme

\section{RESULTS AND DISCUSSION}

The collected dataset has been explored with two standards from ML classifiers. The F-measure score was used for evaluating the performance of each classifier. The collected tweets are partitioned into a training and testing set. The ratio of the data among the training, and testing sets is 8:2.

Tweets were pre-processed by using: tokenization, stop word removal and punctuation, and stemming. Also, part-of-speech (PoS) tags are applying to define distinctive attributes. We focused on selecting the adjectives phrase that can help to interpret the ambiguous words. For filtering the useless word, the negation expressions are considered useful and essential words. Thus, we kept these expressions. In the following tables, the results of the different classifiers with various types of settings are displayed. In Table 2, The original texts were pre-processed by removing stop words. In this setting, the performance of SVM is slightly better than NB with increasing by 0.01 . Table 3 shows a Noticeable change when the PoS tag is used beside removing stop words steps. The performance of NB is increased to 0.80 while SVM is decreased to 0.74 . 
Table 2. Evaluation results of NB and SVM classifier with removing stopwords

\begin{tabular}{ccccc}
\hline Classifier & POS & Stop Word & Stemming & Accuracy \\
\hline NB & 0 & 1 & 0 & 0.77 \\
SVM & 0 & 1 & 0 & .078 \\
\hline
\end{tabular}

Table 3. Evaluation results of NB and SVM classifier with removing stopwords and enable PoS tag

\begin{tabular}{ccccc}
\hline Classifier & POS & Stop Word & Stemming & Accuracy \\
\hline NB & 1 & 1 & 0 & 0.80 \\
SVM & 1 & 1 & 0 & 0.74 \\
\hline
\end{tabular}

The accuracy of both classifies was re-measured but in this time we enabled the stemmer. As shown by Table 4, the worst result is obtain. The performances of NB and SVM are reduced to 0.68 and 0.65 respectively after stemming. The reason for that may be attributed to the role of the stemming technique in lemmatizing words and this may change its emotional means. Also, the stemming operations may not be accurate. After that in Table 5, it can be seen that the performance of SVM was improved once the removing stop words and enable stemming steps are applied. The same case occurred with NB with the same settings.

Table 4. Evaluation results of NB and SVM classifier with stemming

\begin{tabular}{ccccc}
\hline Classifier & POS & Stop Word & Stemming & Accuracy \\
\hline NB & 0 & 0 & 1 & 0.68 \\
SVM & 0 & 0 & 1 & .065 \\
\hline
\end{tabular}

Table 5. Evaluation results of NB and SVM classifier with removing stopwords and stemming

\begin{tabular}{ccccc}
\hline Classifier & POS & Stop Word & Stemming & Accuracy \\
\hline NB & 0 & 1 & 1 & 0.81 \\
SVM & 0 & 1 & 1 & .075 \\
\hline
\end{tabular}

Obviously, the best accuracy reached 0.80 and 0.78 for NB and SVM consequently. NB model showed a slight better performance than the SVM model. This shows that the NB classifier can correctly classify more texts into positive and negative than the SVM classifier. This result is consistent with the results of [54]. However, others found that SVM works better than NB [35], [55], [56]. On the other hand, other declared that both algorithms are equally efficient with a slightly small difference [57].

Typically, Researchers in [54], [58] described the supervised approach as the baseline for sentiment classification. It is found that the supervised learning has a higher efficiency when it is applied on a large textual data than unsupervised learning methods [1], [59]. Also, as reported by [2], [60], the supervised learning approach can classify unknown documents based on the supervised learning method rather than the lexicon method which depends on the deep learning of their resources for categorizing texts.

\section{CONCLUSION}

Communications media are still in progress. This is due to the involving the applications of social media in our daily lifestyle. This motivates different Institutions and governments systems to move towards involving the active part of textual classification in their works to achieve their goals. In the current paper, we presented a model for sentiment analysis of Twitter posts related to the COVID-19 vaccines. This can help the health system and organizations such as World Health Organization (WHO) to use social media like the Twitter and Facebook. Platform to promote the importance of vaccination. Also, understanding individual's opinions concerning vaccination could help to end the pandemic through supporting positive tweets concern vaccines and reject negative ones. We utilized two ML classifiers (NB and SVM) for analyzing the sentiment and opinions of around 15,000 tweets. Experimental results showed that the NB classifier performs outperforms the SVM classifier. The obtained results are promising and encouraging to keep working on this topic by examining more countries and different languages. Also, we plan to imply or combine supervised and unsupervised learning methods to test more models.

\section{REFERENCES}

[1] R. Aguas, R. M. Corder, J. G. King, G. Gonçalves, M. U. Ferreira, and M. M. Gabriela Gomes, "Herd immunity thresholds for SARS-CoV-2 estimated from unfolding epidemics," medRxiv, pp. 1-41, 2020, doi: 10.1101/2020.07.23.20160762. 
[2] E. Cambria, "Affective Computing and Sentiment Analysis," IEEE Intell. Syst., vol. 31, no. 2, 2016, doi: 10.1109/MIS.2016.31.

[3] S. Yousefinaghani, R. Dara, Z. Poljak, T. M. Bernardo, and S. Sharif, "The Assessment of Twitter's Potential for Outbreak Detection: Avian Influenza Case Study,” Sci. Rep., vol. 9, no. 1, pp. 1-17, 2019, doi: 10.1038/s41598019-54388-4.

[4] M. Aldwairi and L. Tawalbeh, "Security techniques for intelligent spam sensing and anomaly detection in online social platforms," Int. J. Electr. Comput. Eng., vol. 10, no. 1, p. 275, Feb. 2020, doi: 10.11591/ijece.v10i1.pp275-287.

[5] P. Mookdarsanit and L. Mookdarsanit, "The covid-19 fake news detection in thai social texts," Bulletin of Electrical Engineering and Informatics (BEEI), vol. 10, no. 2, pp. 988-998, 2021, doi: 10.11591/eei.v10i2.2745.

[6] M. S. Steffens, A. G. Dunn, K. E. Wiley, and J. Leask, "How organisations promoting vaccination respond to misinformation on social media: a qualitative investigation," BMC Public Health, vol. 19, no. 1, pp. 1-12, 2019, doi: 10.1186/s12889-019-7659-3.

[7] E. Bonnevie, A. Gallegos-Jeffrey, J. Goldbarg, B. Byrd, and J. Smyser, "Quantifying the rise of vaccine opposition on Twitter during the COVID-19 pandemic," J. Commun. Healthc., vol. 14, no. 1, pp. 12-19, 2020, doi: $10.1080 / 17538068.2020 .1858222$.

[8] L. Sørensen, "User managed trust in social networking - Comparing Facebook, MySpace and Linkedin," Proc. 2009 1st Int. Conf. Wirel. Commun. Veh. Technol. Inf. Theory Aerosp. Electron. Syst. Technol. Wirel. VITAE 2009, pp. 427-431, 2009, doi: 10.1109/WIRELESSVITAE.2009.5172486.

[9] M. S. M. Suhaimin, M. H. A. Hijazi, R. Alfred, and F. Coenen, "Modified framework for sarcasm detection and classification in sentiment analysis," Indonesian Journal of Electrical Engineering and Computer Science (IJEECS), vol. 13, no. 3, pp. 1175-1183, 2019, doi: 10.11591/ijeecs.v13.i3.pp1175-1183.

[10] A. R. Lubis, M. K. M. Nasution, O. S. Sitompul, and E. M. Zamzami, "The effect of the TF-IDF algorithm in times series in forecasting word on social media," Indonesian Journal of Electrical Engineering and Computer Science (IJEECS), vol. 22, no. 2, p. 976, 2021, doi: 10.11591/ijeecs.v22.i2.pp976-984.

[11] L. Yang, X. Geng, and H. Liao, "A web sentiment analysis method on fuzzy clustering for mobile social media users," Eurasip J. Wirel. Commun. Netw., vol. 2016, no. 1, 2016, doi: 10.1186/s13638-016-0626-0.

[12] N. Genc-Nayebi and A. Abran, "A systematic literature review: Opinion mining studies from mobile app store user reviews,” J. Syst. Softw., vol. 125, pp. 207-219, 2017, doi: 10.1016/j.jss.2016.11.027.

[13] S. Siddiqui, A. A. Monem, and K. Shaalan, "Sentiment analysis in Arabic," Lect. Notes Comput. Sci. (including Subser. Lect. Notes Artif. Intell. Lect. Notes Bioinformatics), vol. 9612, pp. 409-414, 2016, doi: 10.1007/978-3-31941754-7 41.

[14] P. D. Turney, "Thumbs up or thumbs down?,” Proc. 40th Annu. Meet. Assoc. Comput. Linguist., no. July, pp. 417424, 2002, doi: 10.3115/1073083.1073153.

[15] D. H. Abd, A. R. Abbas, and A. T. Sadiq, "Analyzing sentiment system to specify polarity by lexicon-based," Bulletin of Electrical Engineering and Informatics (BEEI), vol. 10, no. 1, pp. 283-289, 2021, doi: 10.11591/eei.v10i1.2471.

[16] C. L. Liu, W. H. Hsaio, C. H. Lee, G. C. Lu, and E. Jou, "Movie rating and review summarization in mobile environment," IEEE Trans. Syst. Man Cybern. Part C Appl. Rev., vol. 42, no. 3, pp. 397-407, 2012, doi: 10.1109/TSMCC.2011.2136334.

[17] Y. Luo and W. Huang, "Product review information extraction based on adjective opinion words," Proc. - 4th Int. Jt. Conf. Comput. Sci. Optim. CSO 2011, pp. 1309-1313, 2011, doi: 10.1109/CSO.2011.209.

[18] R. Liu, R. Xiong, and L. Song, "A sentiment classification method for chinese document," ICCSE 2010 - 5th Int. Conf. Comput. Sci. Educ. Final Progr. B. Abstr., pp. 918-922, 2010, doi: 10.1109/ICCSE.2010.5593462.

[19] A. Khan and B. Baharudin, "Sentiment classification using sentence-level semantic orientation of opinion terms from blogs," 2011 Natl. Postgrad. Conf. - Energy Sustain. Explor. Innov. Minds, NPC 2011, 2011, doi: 10.1109/NatPC.2011.6136319.

[20] L. Ramachandran and E. F. Gehringer, "Automated assessment of review quality using latent semantic analysis," Proc. 2011 11th IEEE Int. Conf. Adv. Learn. Technol. ICALT 2011, pp. 136-138, 2011, doi: 10.1109/ICALT.2011.46.

[21] D. Munandar, A. F. Rozie, and A. Arisal, "A multi domains short message sentiment classification using hybrid neural network architecture," Bulletin of Electrical Engineering and Informatics (BEEI), vol. 10, no. 4, pp. 21812191, 2021, doi: 10.11591/EEI.V10I4.2790.

[22] D. K. Behera, M. Das, S. Swetanisha, and P. K. Sethy, "Hybrid model for movie recommendation system using content K-nearest neighbors and restricted boltzmann machine," Indonesian Journal of Electrical Engineering and Computer Science (IJEECS), vol. 23, no. 1, pp. 445-452, 2021, doi: 10.11591/ijeecs.v23.i1.pp445-452.

[23] A. H. Yassir, A. A. Mohammed, A. A. J. Alkhazraji, M. E. Hameed, M. S. Talib, and M. F. Ali, "Sentimental classification analysis of polarity multi-view textual data using data mining techniques," International Journal of Electrical and Computer Engineering (IJECE), vol. 10, no. 5, pp. 5526-5534, 2020, doi: 10.11591/IJECE.V10I5.PP5526-5534.

[24] M. A. B. W. Nordin, D. Vedenyapin, M. F. Alghifari, and T. S. Gunawan, "The disruptometer: An artificial intelligence algorithm for market insights," Bulletin of Electrical Engineering and Informatics (BEEI), vol. 8, no. 2, pp. 727-734, 2019, doi: 10.11591/eei.v8i2.1494.

[25] M. R. DeVerna et al., "CoVaxxy: A Collection of English-language Twitter Posts About COVID-19 Vaccines," arXiv, 2021, [Online]. Available: http://arxiv.org/abs/2101.07694.

[26] D. Surian, D. Q. Nguyen, G. Kennedy, M. Johnson, E. Coiera, and A. G. Dunn, "Characterizing Twitter discussions about HPV vaccines using topic modeling and community detection," J. Med. Internet Res., vol. 18, no. 8, p. e232, Aug. 2016, doi: 10.2196/jmir.6045. 
[27] G. B.-Orgaz, J. H.-Castro, and D. Camacho, "Detecting discussion communities on vaccination in Twitter," Futur. Gener. Comput. Syst., vol. 66, pp. 125-136, 2016, doi: 10.1016/j.future.2016.06.032.

[28] X. Huang et al., "Examining patterns of influenza vaccination in social media," 2017. [Online]. Available: https://www.cs.jhu.edu/ mdredze/publications/2017_w3phi_vaccines.pdf

[29] M. A. Al-Hagery, M. A. Al-Assaf, and F. M. Al-Kharboush, "Exploration of the best performance method of emotions classification for arabic tweets," Indonesian Journal of Electrical Engineering and Computer Science (IJEECS), vol. 19, no. 2, pp. 1010-1020, 2020, doi: 10.11591/ijeecs.v19.i2.pp1010-1020.

[30] N. P. Shetty, B. Muniyal, A. Anand, S. Kumar and S. Prabhu, "Predicting depression using deep learning and ensemble algorithms on raw Twitter data," International Journal of Electrical and Computer Engineering (IJECE), vol. 10, no. 4, pp. 3751-3756, 2020, doi: 10.11591/ijece.v10i4.pp3751-3756.

[31] R. A. Calvo, S. Member, and S. D. Mello, "Affect Detection: An Interdisciplinary Review of Models, Methods, and Their Applications," 18 IEEE Trans. Affect. Comput., vol. 1, no. 1, pp. 18-37, 2010, doi: 10.1109/TAFFC.2010.1.

[32] A. Batliner, S. Steidl, and D. Seppi, "Recognising realistic emotions and affect in speech : State of the art and lessons learnt from the first challenge," Speech Commun., vol. 53, pp. 1062-1087, 2011, doi: 10.1016/j.specom.2011.01.011.

[33] B. Pang and L. Lee, "Opinion Mining and Sentiment Analysis," Found. Trends_R Inf. Retr., vol. 2, pp. 1-135, 2008, doi: 10.1561/1500000001.

[34] B. Liu, "Sentiment Sentiment Analysis Analysis and and Opinion Opinion Mining Mining," SyntheSiS LectureS on human Language technoLogieS, 2012. doi: 10.2200/S00416ED1V01Y201204HLT016.

[35] S. Raaijmakers, K. Truong, and T. Wilson, "Multimodal subjectivity analysis of multiparty conversation," in EMNLP 2008 - 2008 Conference on Empirical Methods in Natural Language Processing, Proceedings of the Conference: A Meeting of SIGDAT, a Special Interest Group of the ACL, 2008, no. October, pp. 466-474. doi: $10.3115 / 1613715.1613774$

[36] T. Wilson, J. Wiebe, and P. Hoffmann, "Recognizing Contextual Polarity in Phrase-Level Sentiment Analysis," Proc. ofHuman Lang. Technol. Conf. Conf. Empir. Methods Nat. Lang., no. October, pp. 347-354, 2005, doi: $10.3115 / 1220575.1220619$

[37] E. Cambria, S. Poria, R. Bajpai, and B. Schuller, "SenticNet 4: A semantic resource for sentiment analysis based on conceptual primitives," COLING 2016 - 26th Int. Conf. Comput. Linguist. Proc. COLING 2016 Tech. Pap., pp. 2666-2677, 2016.

[38] M. A. Muslim and Y. Dasril, "Company bankruptcy prediction framework based on the most influential features using XGBoost and stacking ensemble learning," International Journal of Electrical and Computer Engineering (IJECE), vol. 11, no. 6, pp. 5549-5557, 2021, doi: 10.11591/ijece.v11i6.pp5549-5557.

[39] S. Yousukkee and N. Wisitpongphan, "Analysis of spammers' behavior on a live streaming chat," IAES Int. J. Artif. Intell., vol. 10, no. 1, pp. 139-150, 2021, doi: 10.11591/ijai.v10.i1.pp139-150.

[40] D. Rajeshwari and S. Vagdevi, "Framework for opinion as a service on review data of customer using semantics based analytics," International Journal of Electrical and Computer Engineering (IJECE), vol. 10, no. 5, pp. 54535461, 2020, doi: 10.11591/IJECE.V10I5.PP5453-5461.

[41] N. S. Shaeeali, A. Mohamed, and S. Mutalib, "Customer reviews analytics on food delivery services in social media: A review," IAES Int. J. Artif. Intell., vol. 9, no. 4, pp. 691-699, 2020, doi: 10.11591/ijai.v9.i4.pp691-699.

[42] I. Chaturvedi and E. Cambria, "Lyapunov Filtering of Objectivity for Spanish Sentiment Model," Int. Jt. Conf. Neural Networks, pp. 4474-4481, 2016, doi: 10.1109/ijenn.2016.7727785.

[43] S. Poria, E. Cambria, and A. Gelbukh, "Aspect extraction for opinion mining with a deep convolutional neural network," Knowledge-Based Syst., vol. 108, pp. 42-49, 2016, doi: 10.1016/j.knosys.2016.06.009.

[44] R. Mihalcea and A. Garimella, "What Men Say, What Women Hear : Finding Gender- Specifi c Meaning Shades," IEEE Intell. Syst., 2016, doi: 10.1111/j.1083-6101.2005.tb00238.x.8.

[45] Y. Ma, E. Cambria, and S. Gao, "Label Embedding for Zero-shot Fine-grained Named Entity Typing," 171 Proc. ofCOLING 2016, 26th Int. Conf. Comput. Linguist. Tech. Pap. pages, pp. 171-180, 2016.

[46] N. M. Samsudin, C. F. B. Mohd Foozy, N. Alias, P. Shamala, N. F. Othman, and W. I. S. Wan Din, "Youtube spam detection framework using naïve bayes and logistic regression," Indonesian Journal of Electrical Engineering and Computer Science (IJEECS), vol. 14, no. 3, pp. 1508-1517, 2019, doi: 10.11591/ijeecs.v14.i3.pp1508-1517.

[47] A. Ashraf, T. S. Gunawan, B. S. Riza, E. V. Haryanto, and Z. Janin, "On the review of image and video-based depression detection using machine learning," Indonesian Journal of Electrical Engineering and Computer Science (IJEECS), vol. 19, no. 3, pp. 1677-1684, 2020, doi: 10.11591/ijeecs.v19.i3.pp1677-1684.

[48] L. P. Morency, R. Mihalcea, and P. Doshi, "Towards multimodal sentiment analysis: Harvesting opinions from the web," in ICMI'11 - Proceedings of the 2011 ACM International Conference on Multimodal Interaction, 2011, pp. 169-176. doi: 10.1145/2070481.2070509.

[49] Y. Zahidi, Y. El Younoussi, and Y. Al-Amrani, "Different valuable tools for Arabic sentiment analysis: a comparative evaluation," International Journal of Electrical and Computer Engineering (IJECE), vol. 11, no. 1, pp. 753-762, 2021, doi: 10.11591/ijece.v11i1.pp753-762.

[50] Y. Zahidi, Y. El Younoussi, and Y. Al-Amrani, "A powerful comparison of deep learning frameworks for Arabic sentiment analysis," International Journal of Electrical and Computer Engineering (IJECE), vol. 11, no. 1, pp. 745-752, 2021, doi: 10.11591/ijece.v11i1.pp745-752.

[51] N. D. Zaki, N. Y. Hashim, Y. M. Mohialden, M. A. Mohammed, T. Sutikno, and A. H. Ali, "A real-time big data sentiment analysis for iraqi tweets using spark streaming," Bulletin of Electrical Engineering and Informatics (BEEI), vol. 9, no. 4, pp. 1411-1419, 2020, doi: 10.11591/eei.v9i4.1897. 
[52] P. P. Rokade and D. A. Kumari, "Business intelligence analytics using sentiment analysis-a survey," International Journal of Electrical and Computer Engineering (IJECE), vol. 9, no. 1, pp. 613-620, 2019, doi: 10.11591/ijece.v9i1.pp613-620.

[53] S. Sun, C. Luo, and J. Chen, "A review of natural language processing techniques for opinion mining systems," Inf. Fusion, vol. 36, pp. 10-25, 2017, doi: 10.1016/j.inffus.2016.10.004.

[54] H. Zhang, W. Gan, and B. Jiang, "Machine Learning and Lexicon based Methods for Sentiment Classification : A Survey 1," th Web Inf. Syst. Appl. Conf. Mach., pp. 262-265, 2014, doi: 10.1109/WISA.2014.55.

[55] H. Zou and X. Tang, "Sentiment Classification Using Machine Learning Techniques with Syntax Features," Int. Conf. Comput. Sci. Comput. Intell. Sentim., 2015, doi: 10.1109/CSCI.2015.44.

[56] E. Sutoyo and A. Almaarif, "Twitter sentiment analysis of the relocation of Indonesia's capital city," Bulletin of Electrical Engineering and Informatics (BEEI), vol. 9, no. 4, pp. 1620-1630, 2020, doi: 10.11591/eei.v9i4.2352.

[57] N. A. Abdulla, N. A. Ahmed, M. A. Shehab, and M. Al-Ayyoub, "Arabic sentiment analysis: Lexicon-based and corpus-based," 2013 IEEE Jordan Conf. Appl. Electr. Eng. Comput. Technol. AEECT 2013, 2013, doi: 10.1109/AEECT.2013.6716448.

[58] R. Bhalla and A. Bagga, "Opinion mining framework using proposed rb-bayes model for text classification," International Journal of Electrical and Computer Engineering (IJECE), vol. 9, no. 1, pp. 477-484, 2019, doi: 10.11591/ijece.v9i1.pp477-484.

[59] C. S. G. Khoo, "Lexicon-based sentiment analysis : Comparative evaluation of six sentiment lexicons," J. Inf. Sci., 2017, doi: 10.1177/0165551517703514.

[60] M. Taboada, J. Brooke, M. Tofiloski, K. Voll, and M. Stede, "Lexicon-Based Methods for Sentiment Analysis DRAFT DRAFT DRAFT!,” Comput. Linguist., vol. 37, no. 2, pp. 267-307, 2011, doi: 10.1162/COLI_a_00049.

\section{BIOGRAPHIES OF AUTHORS}
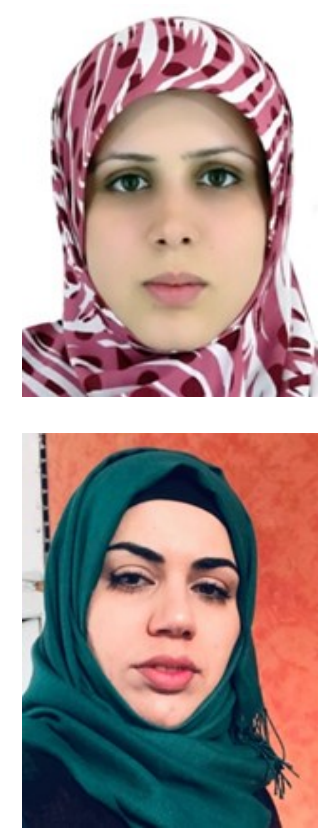

Noralhuda N. Alabid is a lecturer and researcher at the Department of Computer Science, Faculty of Education, University of Kufa (UOK), Najaf, Iraq. She has worked at UOK since 2012. She has an MS in the field of Advanced Computer Science. She graduated from the Department of Computer Science, Faculty of Science, University of Sheffield, UK. Her background is in the field of computer science with special interests in image processing, bioinformatics, NLP, text processing and medical statistics.

Zainab Dalaf Katheeth is a lecturer and researcher at the Department of Computer Science, Faculty of Computer Science and Mathematics, University of Kufa (UOK), Najaf, Iraq. She graduated from the Computer Engineering and information from the University of Mosul for the year 2006 and holds a master's degree in computer engineering from the University of India, MRIU. 